\title{
196G/A of the Brain-Derived Neurotrophic Factor Gene Polymorphisms Predicts Suicidal Behavior in Schizophrenia Patients
}

\author{
Eun-Jeong Kim and Yong-Ku Kim $\bowtie$ \\ Department of Psychiatry, Korea University Ansan Hospital, Ansan, Republic of Korea
}

Objective Brain-derived neurotrophic factor (BDNF) has possible neurobiologic impact on etiology of schizophrenia. We hypothesized that the specific allele or the genotype such as two single nucleotide polymorphisms (SNPs) , 196G/A (rs6265), 11757G/C(rs16917204) is associated with schizophrenia or its clinical features.

Methods 241 normal controls and 157 schizophrenia patients are included. The differences in allele or genotype distribution for the patients and normal controls were analyzed. We also analyzed clinical variables among patients.

Results We found no significant difference in genotype or allele distributions of two studied SNPs between the patient group and the control group. However, history of suicide attempt was relatively higher in patients with genotype with A allele, compared to patients with genotype G/G for 196G/A (p-value=0.045).

Conclusion Our results suggest that it is possible to use BDNF gene allele and genotype as a predictor for suicide attempt in schizophrenia patients. It can help manage the schizophrenia patients regarding suicidal behavior and furthermore, mortality.

Psychiatry Investig 2018;15(7):733-738

Key Words Brain-derived neurotrophic factor, Schizophrenia, Polymorphisms, Suicide.

\section{INTRODUCTION}

Schizophrenia is a psychiatric disorder characterized by delusions, hallucinations, disorganized behaviors and languages. ${ }^{733}$ Treatment response has increased with the use of typical and atypical antipsychotics. However, approximately $20 \%$ to $30 \%$ of patients do not respond to medication and are considered treatment-resistant patients. ${ }^{2,3}$ Understanding the genetics and neurobiological basis of schizophrenia and characteristics of each patients is necessary for improved treatment responses.

Researchers have attempted to discover the genetic basis of psychiatric disorders, including schizophrenia. Brain-derived

\footnotetext{
Received: January 28, 2018 Revised: February 19, 2018

Accepted: February 27, 2018

$\triangle$ Correspondence: Yong-Ku Kim, MD, PhD

Department of Psychiatry, Korea University Ansan Hospital, 87 Guryong-ro, Sangnok-gu, Ansan 15324, Republic of Korea

Tel: +82-31-412-5140, Fax: +82-31-412-5144, E-mail: yongku@korea.edu

(a) This is an Open Access article distributed under the terms of the Creative Commons Attribution Non-Commercial License (http://creativecommons.org/licenses/bync/4.0) which permits unrestricted non-commercial use, distribution, and reproduction in any medium, provided the original work is properly cited.
}

neurotrophic factor (BDNF) is a highly expressed neurotrophin in the prefrontal cortex and hippocampus. ${ }^{4}$ BDNF has longterm effects on neuronal survival, differentiation, synaptic plasticity, ${ }^{5}$ development of the central nervous system (CNS) in children and maintenance of the CNS in adults. ${ }^{6}$

Whether BDNF is associated with schizophrenia has been investigated ${ }^{7}$ and many models considering such association have been proposed. BDNF modulates the expression of dopamine D3 receptors ${ }^{8}$ and contributes to maturation and plasticity of dopaminergic pathways, including the mesolimbic pathway. ${ }^{9}$ BDNF modulates the firing rates of serotonergic neurons within the raphe nuclei ${ }^{10}$ and this relationship with serotonergic signaling contributes to schizophrenia.

Different effects of single nucleotide polymorphism (SNP) on BDNF have been studied. One of the most studied SNPs, 196G/A (Val66Met, rs6265), plays a modulating role in BDNF activity. SNP 196G/A also showed an association with cognitive impairment and brain volume differences in human studies. ${ }^{11,12}$ Another well-studied SNP, 11757G/C (rs16917204), was associated with Alzheimer's disease with comorbid depression. ${ }^{13,14}$ Many studies from different groups proposed 
SNP 11757G/C was associated with various psychiatric disorders, although consensus has not been reached.

\section{Aims of the study}

We hypothesized specific BDNF gene polymorphisms contribute to susceptibility to schizophrenia. We also hypothesized specific BDNF gene allele and genotype may have relationship with specific clinical characteristics of each patients. Discovering any associations will help the understanding of the neurobiological background of schizophrenia and provide newer treatment options.

\section{METHODS}

A total of 157 patients diagnosed with schizophrenia based on the Diagnostic and Statistical Manual of Mental Disorders, fourth edition (DSM-IV) were enrolled in the present study. We recruited patients who admitted Korea University Ansan Hospital due to acute psychotic symptoms from 2006 to 2011. Each patient received a Structured Clinical Interview for DSM-IV Axis I Disorders (SCID-I) ${ }^{15}$ performed by trained psychiatrists. Subjects with comorbid psychiatric disorders, such as mood disorder, anxiety disorder, neurocognitive disorder or schizoaffective disorder were excluded.

Patient clinical data and past personal history were assessed. Demographic data including age at onset, number of admissions and disease duration, history of suicide attempts were collected. For clinical assessment, severity of disease was evaluated by trained psychiatrists using the Positive And Negative Syndrome Scale (PANSS) ${ }^{16}$ Brief Psychiatric Rating Scale (BPRS) ${ }^{17}$ and Korean version of the Calgary Depression Scale for Schizophrenia (K-CDSS). ${ }^{18}$

The normal control group included 241 healthy individuals who visited Korea University Ansan Hospital for regular health checkups. Subjects with a history of personal or familial psychiatric diagnoses, or who had a Beck Depression Inventory (BDI) score higher than $10^{19}$ or State-Trait Anxiety Inventory (STAI) higher than 40 were excluded. ${ }^{20}$ Written informed consent was obtained from all subjects. The study protocol was approved by the Ethics Committee of Korea University Ansan Hospital (2005AS0008).

DNA analyses and genotyping were performed for both patients and controls. We examined 2 BDNF SNPs, 196G/A (rs6265), and 11757G/C (rs16917204). The genotyping process was performed as follows:

For 196A site, DNA was extracted from blood leukocytes using a commercial DNA extract kit, Wizard Genomic DNA purification kit (Promega, WI, USA). Polymerase chain reaction (PCR) was performed with the forward primer 5'-GAG GCT TGA CAT CAT TGG CT-3' and the reverse primer 5'-
CGT GTA CAA GTC TGC GTC CT-3'. The amplification mixture contained $0.5 \mathrm{uL}$ of $100 \mathrm{ng} / \mathrm{uL}$ DNA, $2.5 \mathrm{uL}$ of $10 \times$ Taq buffer, $0.5 \mathrm{uL}$ of $10 \mathrm{mM}$ dNTP mixture, $1 \mathrm{uL}$ primers, $19.375 \mathrm{uL}$ distilled water and $0.125 \mathrm{uL}$ Taq DNA polymerase (SolGent, Seoul, Korea).

Samples were amplified using a Thermocycler (Veriti 96well thermal cycler, Applied Biosystems) for 35 cycles. After an initial $10 \mathrm{~min}$ at $95^{\circ} \mathrm{C}$, each cycle consisted of $30 \mathrm{sec}$ at $94^{\circ} \mathrm{C}, 30 \mathrm{sec}$ at $62^{\circ} \mathrm{C}$ and $30 \mathrm{sec}$ at $72^{\circ} \mathrm{C}$. After a final $5 \mathrm{~min}$ at $72^{\circ} \mathrm{C}$, the reaction was terminated at $4^{\circ} \mathrm{C}$. The amplified DNA was digested with the restriction enzyme NIaIII (New England Biolabs, MA, USA), which cuts at the 196A site, and the product was electrophoresed in 3\% agarose gels and stained with ethidium bromide. Homozygous genotypes were identified based on the presence of $113 \mathrm{bp}$ bands (G/G) or 75 and 38 bp bands (A/A). The heterozygous genotype had the following 3 bands: 113, 75 and 38 bp (G/A). PCR was performed with the forward primer 5 '-CCT CCT GCA GCC ATT AGT3 ' and the reverse primer 5'-AAT ACA AGT AGG ACC CTA GC-3'. The amplification mixture contained $0.5 \mathrm{uL}$ of 100 ng/uL DNA, $2.5 \mathrm{uL}$ of $10 \times$ Taq buffer, $0.5 \mathrm{uL}$ of $10 \mathrm{mM}$ dNTP mixture, $1 \mathrm{uL}$ primers, $19.375 \mathrm{uL}$ distilled water and $0.125 \mathrm{uL}$ Taq DNA polymerase (SolGent).

For $11757 \mathrm{C}$ site, PCR was performed with the forward primer 5'-CCT CCT GCA GCC ATT AGT-3' and the reverse primer 5'-AAT ACA AGT AGG ACC CTA GC-3'. The amplification mixture contained $0.5 \mathrm{uL}$ of $100 \mathrm{ng} / \mathrm{uL}$ DNA, $2.5 \mathrm{uL}$ of $10 \times$ Taq buffer, $0.5 \mathrm{uL}$ of $10 \mathrm{mM}$ dNTP mixture, 1 $\mathrm{uL}$ primers, $19.375 \mathrm{uL}$ distilled water, and $0.125 \mathrm{uL}$ Taq DNA polymerase (SolGent).

Samples were amplified using a Thermocycler (Veriti 96well thermal cycler, Applied Biosystems) for 35 cycles. After an initial $10 \mathrm{~min}$ at $95^{\circ} \mathrm{C}$, each cycle consisted of $30 \mathrm{sec}$ at $94^{\circ} \mathrm{C}, 30 \mathrm{sec}$ at $58^{\circ} \mathrm{C}$ and $30 \mathrm{sec}$ at $72^{\circ} \mathrm{C}$. After a final $5 \mathrm{~min}$ at $72^{\circ} \mathrm{C}$, the reaction was terminated at $4^{\circ} \mathrm{C}$. The amplified DNA was digested with the restriction enzyme AvaII (New England Biolabs), which cuts at the $11757 \mathrm{C}$ site, and the product was electrophoresed in $3.5 \%$ agarose gels and stained with ethidium bromide. Homozygous genotypes were identified based on the presence of 65,61 , and $44 \mathrm{bp}$ bands $(\mathrm{C} / \mathrm{C})$ or 105 and 65 bp bands (G/G). The heterozygous genotype had the following 4 bands: 105, 65, 61, and $44 \mathrm{bp}(\mathrm{C} / \mathrm{G})$.

The demographic data were compared between patient and control groups using $\chi^{2}$ and independent t-tests. For each SNP, whether genotype distribution followed the Hardy-Weinberg equilibrium was tested using the $\chi^{2}$ test. The genotype and allele frequencies of patients and controls were analyzed using $\chi^{2}$ tests. One-way ANOVA was used for comparison of each SNP genotype and clinical scale, clinical variables. Statistical analysis was performed using SPSS version 
21.0 for Windows (IBM Corp., Armonk, NY, USA). Statistical significance level was set at $\mathrm{p}<0.05$ for all statistical analyses.

\section{RESULTS}

We compared demographic data between schizophrenia patients (68 males and 89 females) and healthy controls (121 males and 120 females). The mean age in the patient group was 33.2 years with a standard deviation (SD) of 10.0 years and mean age in the control group was 31.7 years with an SD of 8.4 years. There were no significant gender or age distribution differences between the patient and control groups.

In patients with schizophrenia, the mean age at disease onset was 27.3 years (SD, 9.8 years) and mean number of admissions was 1.3 (SD, 1.9). Mean duration of disease was 72.1 months (SD, 77.3 months).

The distribution of BDNF 196G/A (rs6265), and 11757G/ C (rs16917204) polymorphisms followed the Hardy-Weinberg equilibrium. The Hardy-Weinberg equilibrium values for schizophrenia patients for BDNF 196G/A were $\chi^{2}=3.1408$, $\mathrm{df}=1, \mathrm{p}=0.07$ and of controls $\chi^{2}=0.3678, \mathrm{df}=1, \mathrm{p}=0.5442$. For BDNF $11757 \mathrm{G} / \mathrm{C}$, the equilibrium values for schizophrenia patients were $\chi^{2}=0.0534, \mathrm{df}=1, \mathrm{p}=0.8172$ and of controls $\chi^{2}=2.0874, \mathrm{df}=1, \mathrm{p}=0.1485$.

The genotype and allele frequencies for the 2 SNPs were not different between patients and controls (Table 1).

We studied the clinical variables, genotype and allele distributions among schizophrenia patients. Associations be- tween the assessed scales and genotype distribution were not found. For 196G/A, 3 different genotypes had a mean total PANSS score ranging from 96.455 to 97.268 , showing no group differences. For further evaluation, we divided total PANSS score by subscales, positive, negative, general psychopathology each. For 196G/A, mean positive score for each genotype ranged from 24.391 to 25.929 . Mean negative subscale score ranged from 22.434 to 23.391 , and mean general psychopathology subscale ranged from 48.171 to 48.268 . For $11757 \mathrm{G} / \mathrm{C}$, no group difference was found among positive, negative, general psychopathology subscales. All those assessed scales and genotype distribution did not show any differences among genotype groups. For both K-CDSS and BPRS, no difference was found among each genotype of 196G/A. For 11757G/C genotype groups, both K-CDSS and BPRS showed no group differences.

Another clinical variable, number of admission and total duration of disease were also assessed. For 196G/A and $11757 \mathrm{G} / \mathrm{C}$, mean number of admission ranged from 0.593 to 1.477 and 1.265 to 1.286 , each. For mean total duration of disease, it ranged from 39.235 to 88.309 months and from 51.0 to 77.172 months for $196 \mathrm{G} / \mathrm{A}$ and $11757 \mathrm{G} / \mathrm{C}$, each. There was no group difference for both number of admission and total duration of disease.

There were no BDNF 196G/A or 11757G/C allele or genotype differences between subjects with or without a previous suicide attempt, with the following exception: there was a higher frequency of suicide attempts among patients car-

Table 1. Genotype and allele frequencies of BDNF SNPs 196G/A, and 11757G/C in schizophrenia patients and normal controls

\begin{tabular}{|c|c|c|c|c|c|c|c|c|c|}
\hline \multirow[b]{2}{*}{ 196G/A } & \multicolumn{3}{|c|}{ Genotype } & \multirow{2}{*}{$\frac{\chi^{2}}{1.010}$} & \multirow{2}{*}{$\frac{p}{0.603^{*}}$} & \multicolumn{2}{|c|}{ Allele } & \multirow{2}{*}{$\frac{\chi^{2}}{0.00}$} & \multirow{2}{*}{$\frac{p}{0.989^{*}}$} \\
\hline & $\mathrm{G} / \mathrm{G}$ & G/A & $\mathrm{A} / \mathrm{A}$ & & & G & A & & \\
\hline Patients & 45 & 88 & 24 & & & 178 & 136 & & \\
\hline Controls & 75 & 123 & 43 & & & 273 & 209 & & \\
\hline $11757 \mathrm{G} / \mathrm{C}$ & $\mathrm{G} / \mathrm{G}$ & $\mathrm{G} / \mathrm{C}$ & $\mathrm{C} / \mathrm{C}$ & 1.171 & $0.557^{*}$ & G & $\mathrm{C}$ & 0.736 & $0.391^{*}$ \\
\hline Patients & 65 & 71 & 21 & & & 201 & 113 & & \\
\hline Controls & 95 & 104 & 42 & & & 294 & 188 & & \\
\hline
\end{tabular}

*for genotype and allele frequencies of both 2 SNPs, no statistically different distribution was found

Table 2. History of suicide attempts by genotype and allele frequencies in schizophrenia patients when dividing genotypes based on the existence of the specific allele

\begin{tabular}{|c|c|c|c|c|}
\hline & \multicolumn{2}{|c|}{ Genotype } & \multirow{2}{*}{$\frac{\chi^{2}}{4.037}$} & \multirow{2}{*}{$\frac{p}{0.045^{\prime}}$} \\
\hline 196G/A & Patients without $A$ allele & Patients with A allele & & \\
\hline Suicide attempt & 3 & 22 & & \\
\hline None & 42 & 90 & & \\
\hline $11757 \mathrm{G} / \mathrm{C}$ & Patients without $\mathrm{C}$ allele & Patients with $\mathrm{C}$ allele & 0.024 & 0.877 \\
\hline Suicide attempt & 10 & 15 & & \\
\hline None & 55 & 77 & & \\
\hline
\end{tabular}

${ }^{*}$ genotypes which included the A allele, G/A and A/A, showed a statistically higher history of previous suicide attempts 
rying the 196G/A A allele (196G/A heterozygotes and 196 A/A homozygotes), than carriers of the $196 \mathrm{G} / \mathrm{G}$ genotype (Table 2).

\section{DISCUSSION}

Many researchers have attempted to determine correlations between psychiatric disorders and BDNF SNP genotypes; however, results among different disorders were inconsistent. A Scottish study enrolled 320 probands diagnosed with schizophrenia and 350 normal controls. Allele G of 196G/A showed strong association with schizophrenia but not bipolar disorder, compared to normal controls. ${ }^{21} \mathrm{Con}$ versely, a Asian study showed negative results; the G/G, G/A and A/A genotype distribution of 196G/A was not different between schizophrenia patient group and healthy control groups $(\mathrm{p}=0.31){ }^{22}$ Regarding $11757 \mathrm{G} / \mathrm{C}$, no allele or genotype difference was found when comparing schizophrenia patients with normal controls.?

In this study, we compared genotype distribution differences for 2 SNPs, 196G/A and 11757G/C between schizophrenia patients and controls. For genotype and allele distributions, no significant association with schizophrenia was found. Our results were in agreement with the Dutch study and other previous studies. ${ }^{7,23,24}$ The results were consistent among the studies indicating BDNF SNP genotyping alone cannot predict susceptibility of schizophrenia.

Theoretical bases of how different SNPs affect pathophysiology of psychiatric disorders are not well established. However, considering preexisting results will help understand our results. 196G/A is known to modulate expression of BDNF protein. Structurally, 196G/A allele difference results in different brain volumes and A allele in smaller prefrontal, dorsolateral volumes compared to G allele. $.^{25} 11757 \mathrm{G} / \mathrm{C}$ is also associated with structural differences, frontal gray matter volume variation, and cerebellar hemisphere white and gray matter volumes in imaging studies. Considering these results, susceptibility of schizophrenia depending on SNP difference can be explained partially. 11757G/C allele variance, for example, results in difference in frontal volume, especially in smaller frontal volume, and this hypofrontality also contributes to schizophrenia. A few evidence for impact of different SNPs in brain volume or BDNF level exist, however, it was not a distinct feature in etiology of schizophrenia.

SNP genotypes could not predict clinical severity in schizophrenia patients. In our study, the 2 SNP genotypes did not show significant differences in symptom severity scales. This result is in agreement with a previous study showing that 196G/A genotype is related with the presence of symptoms rather than severity of symptoms. ${ }^{6}$ Affective symptoms, anxi- ety and depressive symptoms showed an association with carrying the A allele. ${ }^{26}$ However, a previous study showed the G/A genotype for 196G/A was related to earlier onset of disease and the G/G genotype was associated with severe symptoms. ${ }^{27}$ Future studies are needed to clarify such inconsistent results.

In the present study, the existence of A allele in 196G/A correlated with a personal history of suicide attempts. Both postmortem study ${ }^{28}$ and study with living patients ${ }^{29}$ showed the presence of the A allele was associated with susceptibility to suicide in depressed patients. In the present study, the A allele also predicted suicide attempts in schizophrenia patients regardless of different diagnoses.

Considering function of SNP 196G/A in BDNF expression may help understand our results. As mentioned above, 196G/A expression plays a role in modulating production and secretion of BDNF protein in brain. Especially, A allele in 196G/A is related to lowering production of $\mathrm{BDNF}$ in brain compared to G allele..$^{30}$ Low BDNF level causes disruption in the neuronal survival and plasticity of serotonergic and dopaminergic neurons, and, such disruption can result in suicidality. ${ }^{31}$ Low brain BDNF level in hippocampus and prefrontal cortex is also shown to be related with suicidal behavior. ${ }^{29}$ Recently, suicide-attempted patients had lower levels of BDNF, even in serum. ${ }^{32}$ Furthermore, A allele in 196G/A was also dominant among patients with anxiety-related personality traits, and it brings higher frequency of suicidal behaviors. ${ }^{29}$ All these impacts of BDNF protein expression on neurobiological pathways and personality traits would explain specific BDNF SNP and its relation to suicide behaviors.

Previous studies also confirm our results. A allele in 196G/ $\mathrm{A}$ and its relation with the suicide behavior has been well studied. A/A seemed to be the most related with history of suicide compared to G/A or G/G. ${ }^{33}$ Like our study, A carrier showed relation with suicide attempt in schizophrenia patients. Among A carriers, previous history of childhood trauma or stressful life events even raised the risk for suicide attempt. ${ }^{28}$ Psychiatric-related dysfunction in the patients also raised risk for suicide attempt among A carriers. ${ }^{34}$

Suicide attempt rate ranges from $18 \%$ to $55 \%$ among schizophrenia patients. ${ }^{35}$ Similar to many other risk factors, such as younger age, ${ }^{36}$ male gender, ${ }^{37}$ earlier age at onset, ${ }^{38}$ being unmarried, ${ }^{39}$ presence of the A allele can be used as risk factors for suicide in schizophrenia patients. Verifying susceptibility to suicide in a clinical setting will help manage these patients which could be life-saving.

Overall results also suggest that BDNF gene polymorphism cannot directly predict susceptibility of disease, but rather predict the specific symptom entity among psychiatric disorders. A allele in 196G/A and its relation with affective 
symptoms in schizophrenia ${ }^{26}$ for instance. In our study, we also found that the BDNF gene polymorphism could not predict susceptibility to the disease itself, but rather predict relation with specific clinical features among schizophrenia patients. Similar results were suggested in study of the depression. ${ }^{40}$ It was also found that specific alleles and genotypes were related with severe depressive symptoms, rather than diagnosis of depression. These results can partly explain that the BDNF polymorphism is specifically related with the neuropsychiatric symptoms. Further study to support current result is required.

This study had several limitations. The number of patients and controls was relatively small. Also, our results do not include quantitative information about the BDNF level in both each brain sites and whole body serum, but only genotypes and gene alleles. Few of our analysis didn't have done corrections for multiple comparisons. Lastly, our study included only Korean patients and limited ethnic diversity could have produced biased results. Regardless of these limitations, this study is valuable for examining clinical variables and their relationship with BDNF SNP genotype. Future studies overcoming our study limitations will expand the understanding of the neurobiology and etiology of schizophrenia.

\section{Acknowledgments}

This paper was supported by Korea University.

\section{REFERENCES}

1. American Psychiatric Association. Diagnostic and statistical manual of mental disorders (DSM-5®). Washington DC: American Psychiatric Pub; 2013.

2. Kane J, Honigfeld G, Singer J, Meltzer H. Clozapine for the treatmentresistant schizophrenic: a double-blind comparison with chlorpromazine. Arch Gen Psychiatry 1988;45:789-796.

3. Elkis H. Treatment-resistant schizophrenia. Psychiatr Clin North Am 2007;30:511-533.

4. Liu X, Ernfors P, Wu H, Jaenisch R. Sensory but not motor neuron deficits in mice lacking NT4 and BDNF. Nature 1995;375:238-241.

5. Riccio A, Ahn S, Davenport CM, Blendy JA, Ginty DD. Mediation by a CREB family transcription factor of NGF-dependent survival of sympathetic neurons. Science 1999;286:2358-2361.

6. Notaras M, Hill R, Van den Buuse M. A role for the BDNF gene Val66 Met polymorphism in schizophrenia? A comprehensive review. Neurosci Biobehav Rev 2015;51:15-30.

7. Jönsson EG, Edman-Ahlbom B, Sillën A, Gunnar A, Kulle B, Frigessi $A$, et al. Brain-derived neurotrophic factor gene (BDNF) variants and schizophrenia: an association study. Prog Neuropsychopharmacol Biol Psychiatry 2006;30:924-933.

8. Guillin O, Diaz J, Carroll P, Griffon N, Schwartz JC, Sokoloff P. BDNF controls dopamine D3 receptor expression and triggers behavioural sensitization. Nature 2001;411:86-89.

9. Baker SA, Stanford LE, Brown RE, Hagg T. Maturation but not survival of dopaminergic nigrostriatal neurons is affected in developing and aging BDNF-deficient mice. Brain Res 2005;1039:177-188.

10. Celada P, Siuciak JA, Tran TM, Altar CA, Tepper JM. Local infusion of brain-derived neurotrophic factor modifies the firing pattern of dorsal raphe serotonergic neurons. Brain Res 1996;712:293-298.
11. Egan MF, Kojima M, Callicott JH, Goldberg TE, Kolachana BS, Bertolino A, et al. The BDNF val66met polymorphism affects activity-dependent secretion of BDNF and human memory and hippocampal function. Cell 2003;112:257-269.

12. Gratacòs M, González JR, Mercader JM, de Cid R, Urretavizcaya M, Estivill X. Brain-derived neurotrophic factor Val66Met and psychiatric disorders: meta-analysis of case-control studies confirm association to substance-related disorders, eating disorders, and schizophrenia. Biol Psychiatry 2007;61:911-922.

13. Kleim JA, Chan S, Pringle E, Schallert K, Procaccio V, Jimenez R, et al. BDNF val66met polymorphism is associated with modified experience-dependent plasticity in human motor cortex. Nature Neurosci 2006;9:735-737.

14. Borroni B, Grassi M, Archetti S, Costanzi C, Bianchi M, Caimi L, et al. BDNF genetic variations increase the risk of Alzheimer's disease-related depression. J Alzheimers Dis 2009;18:867-875.

15. First MB, Spitzer RL, Gibbon M, Williams JB. Structured Clinical Interview for DSM-IV Axis I Disorders. New York: New York State Psychiatric Institute; 1995.

16. Kay SR, Flszbein A, Opfer LA. The positive and negative syndrome scale (PANSS) for schizophrenia. Schizophr Bull 1987;13:261-276.

17. Overall JE, Gorham DR. The brief psychiatric rating scale. Psychol Rep 1962;10:799-812.

18. Kim YK, Won SD, Lee KM, Choi HS, Jang HS, Lee BH, et al. A study on the reliability and validity of the Korean version of the Calgary Depression Scale for Schizophrenia (K-CDSS). J Korean Neuropsychiatr Assoc 2005;44:446-455.

19. Beck AT, Ward CH, Mendelson M, Mock J, Erbaugh J. An inventory for measuring depression. Arch Gen Psychiatry 1961;4:561-571.

20. Spielberger CD. Manual for the State-Trait Anxiety Inventory STAI (form Y)("Self-Evaluation Questionnaire"). Palo Alto, CA: Consulting Psychologists Press, Inc; 1983.

21. Neves-Pereira M, Cheung J, Pasdar A, Zhang F, Breen G, Yates P, et al. BDNF gene is a risk factor for schizophrenia in a Scottish population. Mol Psychiatry 2005;10:208-212.

22. Naoe Y, Shinkai T, Hori H, Fukunaka Y, Utsunomiya K, Sakata S, et al. No association between the brain-derived neurotrophic factor (BDNF) Val66Met polymorphism and schizophrenia in Asian populations: evidence from a case-control study and meta-analysis. Neurosci Lett 2007;415:108-112.

23. Nanko S, Kunugi H, Hirasawa H, Kato N, Nabika T, Kobayashi S. Brain-derived neurotrophic factor gene and schizophrenia: polymorphism screening and association analysis. Schizophr Res 2003;62:281283.

24. Hong CJ, Yu YW, Lin CH, Tsai SJ. An association study of a brain-derived neurotrophic factor Val66Met polymorphism and clozapine response of schizophrenic patients. Neurosci lett 2003;349:206-208.

25. Agartz I, Sedvall GC, Terenius L, Kulle B, Frigessi A, Hall H, et al. BDNF gene variants and brain morphology in schizophrenia. Am J Med Genet B Neuropsychiatr Genet 2006;141:513-523.

26. Sun MM, Yang LM, Wang Y, Feng X, Cui KY, Liu LF, et al. BDNF Val66Met polymorphism and anxiety/depression symptoms in schizophrenia in a Chinese Han population. Psychiatr Genet 2013;23:124129.

27. Suchanek R, Owczarek A, Paul-Samojedny M, Kowalczyk M, Kucia K, Kowalski J. BDNF val66met polymorphism is associated with age at onset and intensity of symptoms of paranoid schizophrenia in a Polish population. J Neuropsychiatry Clin Neurosci 2013;25:88-94.

28. Pregelj P, Nedic G, Paska AV, Zupanc T, Nikolac M, Balažic J, et al. The association between brain-derived neurotrophic factor polymorphism (BDNF Val66Met) and suicide. J Affect Disord 2011;128:287-290.

29. Sarchiapone M, Carli V, Roy A, Iacoviello L, Cuomo C, Latella MC, et al. Association of polymorphism (Val66Met) of brain-derived neurotrophic factor with suicide attempts in depressed patients. Neuropsychobiology 2008;57:139-145. 
30. Hashimoto K. BDNF variant linked to anxiety-related behaviors. Bioessays 2007;29:116-119.

31. Berton O, McClung CA, DiLeone RJ, Krishnan V, Renthal W, Russo $\mathrm{SJ}$, et al. Essential role of BDNF in the mesolimbic dopamine pathway in social defeat stress. Science 2006;311:864-868.

32. Deveci A, Aydemir O, Taskin O, Taneli F, Esen-Danaci A. Serum BDNF levels in suicide attempters related to psychosocial stressors: a comparative study with depression. Neuropsychobiology 2007;56:93-97.

33. Huang TL, Lee C. Associations between brain-derived neurotrophic factor G196A gene polymorphism and clinical phenotypes in schizophrenia patients. Chang Gung Med J 2007;30:408-413.

34. Iga JI, Ueno SI, Yamauchi K, Numata S, Tayoshi-Shibuya S, Kinouchi S, et al. The Val66Met polymorphism of the brain-derived neurotrophic factor gene is associated with psychotic feature and suicidal behavior in Japanese major depressive patients. Am J Med Genet B Neuropsychiatr Genet 2007;144B:1003-1006.
35. Siris SG. Suicide and schizophrenia. J Psychopharmacol 2001;15:127-135.

36. Krausz M, Müller-Thomsen T, Haasen C. Suicide among schizophrenic adolescents in the long-term course of illness. Psychopathology 1995;28:95-103

37. Tsuang MT. Suicide in schizophrenics, manics, depressives, and surgical controls: a comparison with general population suicide mortality. Arch Gen Psychiatry 1978;35:153-155.

38. Gupta S, Black DW, Arndt S, Hubbard WC, Andreasen NC. Factors associated with suicide attempts among patients with schizophrenia. Psychiatr Serv 1998;49:1353-1355.

39. Westermeyer JF, Harrow M, Marengo JT. Risk for suicide in schizophrenia and other psychotic and nonpsychotic disorders. J Nerv Ment Dis 1991;179:259-266.

40. Duncan LE, Hutchison KE, Carey G, Craighead WE. Variation in brain-derived neurotrophic factor (BDNF) gene is associated with symptoms of depression. J Affect Disord 2009;115:215-219. 